\title{
FEASt: A Randomized Controlled Trial for Dysphagia Recovery After an Acute Ischemic Stroke
}

\section{Sandeep Kumar ( $\nabla$ skumar@bidmc.harvard.edu )}

Beth Israel Deaconess Medical Center

\section{Sarah Marchina}

Beth Israel Deaconess Medical Center

\section{Susan Langmore}

Boston University School of Medicine

Joseph Massaro

Boston University School of Public Health

Joseph Palmisano

Boston University School of Public Health

\section{Na Wang}

Boston University School of Public Health

\section{David Eric Searls}

Beth Israel Deaconess Medical Center

\section{Vasileios Lioutas}

Beth Israel Deaconess Medical Center

Jessica Pisegna

Boston University School of Medicine

Cynthia Wagner

Beth Israel Deaconess Medical Center

\section{Anant Shinde}

Beth Israel Deaconess Medical Center

Gottfried Schlaug

Beth Israel Deaconess Medical Center

\section{Research Article}

Keywords: Post-stroke dysphagia, transcranial direct current stimulation, neuroplasticity, randomized clinical trial

Posted Date: March 3rd, 2022

DOI: https://doi.org/10.21203/rs.3.rs-1344219/v1

License: (a) (1) This work is licensed under a Creative Commons Attribution 4.0 International License. Read Full License 


\section{Abstract}

Background: Dysphagia is a serious stroke complication but lacks effective therapy. We investigated safety and preliminary efficacy of anodal transcranial direct current stimulation (atDCS) paired with swallowing exercises in improving post-stroke dysphagia from an acute unilateral hemispheric infarction (UHI).

Methods: Double-blind, early phase-2 randomized controlled trial, in subjects $(n=42)$ with moderate-severe dysphagia [Penetration and Aspiration Scale (PAS) score $\geq 4$ ], from an acute-subacute UHI. Subjects were randomized to Low-Dose, High-Dose atDCS or Sham stimulation for 5 consecutive days. Primary safety outcomes were incidence of seizures, neurological, motor, or swallowing function deterioration. Primary efficacy outcome was a change in PAS scores at day-5 of intervention. Main secondary outcome was dietary improvement at 1-month, assessed by Functional Oral Intake (FOIS) score.

Results: No differences in pre-defined safety outcomes or adjusted mean changes in PAS, FOIS scores, between groups, were observed. Post-hoc analysis demonstrated that 22 / 24 subjects in the combined atDCS group had a clinically meaningful dietary improvement (FOIS score $\geq 5$ ) compared to $8 / 14$ in Sham ( $p=0.037$, Fisher-exact).

Conclusion: atDCS application in the acute-subacute stroke phase is safe but did not decrease risk of aspiration in this early phase trial. The observed dietary improvement is promising and merits further investigation.

\section{Introduction}

Dysphagia is a very common complication of a stroke and carries major implications for stroke survivors [1-3]. It independently increases the risk of pneumonia and malnutrition, promotes early stroke mortality and institutionalization $[1,2,4,5]$. Treatments that help restore normal swallowing can therefore improve stroke outcomes. Unfortunately, such therapies are currently lacking and management usually revolves around providing nutritional support with nasogastric or percutaneous endoscopic gastrostomy tubes until swallowing recovers spontaneously, if at all [6]; patients fed via these means remain vulnerable to serious medical complications and have been observed to have high mortality rates $[7,8]$

In traditional conceptualization of swallowing control, swallowing is regarded solely as a "reflex" that is controlled by the brainstem "swallowing-centers". These models for swallowing regulation, however, do not explain the great versatility of swallowing behaviors in health, that range from reflex swallowing to volitional eating or a combination of volitional and reflexive swallowing such as brisk drinking. Neither do they account for the high incidence of persistent dysphagia observed with isolated hemispheric strokes that spare brainstem structures $[2,3,9]$.

Recent insights on the neurophysiology of swallowing control in health and the neuroplastic processes that drive swallowing recovery after stroke provide a basis to develop and test targeted therapies for post-stroke dysphagia. Studies that are more contemporary show that there is an intricate control of normal swallowing mediated by both cerebral hemispheres, the subcortical regions and the central pattern generators in the medullary pontine regions $[10,11]$. Separate lines of investigations reveal that while unilateral hemisphere infarction can produce dysphagia by damaging the swallowing motor cortex or its projections, the contralateral healthy swallowing cortex possesses the capacity to reorganize sufficiently to enable restoration of normal swallowing [12-14]. The presence of inherent redundancies in swallowing control, where projections from both swallowing cortices converge on a common pool of brainstem structures ("swallowing-centers"), can enable independent unilateral hemispheric regulation of bilateral "swallowing-centers" [15]. These unique features make the undamaged swallowing cortex an attractive 
target for cortical modulation techniques to augment the naturally occurring neuroplastic changes within this region and to enhance its role as a mediator of recovery $[16,17]$.

As hemispheric strokes are the commonest stroke sub-type in the population, they likely contribute disproportionately to the overall dysphagia burden $[2,9,18]$. A non-invasive brain stimulation technique, like transcranial direct current stimulation (tDCS) may be especially well suited to improve swallowing in this stroke population [2, 9]. It has been demonstrated that recovery of swallowing functions in patients with hemispheric lesions occurs via compensatory reorganization of the undamaged cerebral hemisphere and shows a predictable pattern of expansion of the pharyngeal representation in an anterolateral direction, irrespective of lesion site or laterality [14]. This reorganization is preceded by an increase in excitability of the swallowing cortex in the undamaged hemisphere suggesting that the excitability changes drive cortical reorganization [14]. We therefore hypothesize that with hemispheric lesions, where the brainstem and peripheral structures are intact but the upper echelons of the swallowing apparatus are dysfunctional, a cortical stimulation technique can be effective by engaging the intact swallowing motor cortex and helping to restore swallowing functions.

We and others have previously reported on the effect of anodal tDCS (atDCS) for dysphagia recovery after a stroke in pilot studies $[19,20]$. Building upon these experiences, we systematically examined the safety and feasibility, and performed a preliminary assessment of efficacy of atDCS paired with swallowing exercises in improving swallowing functions after an acute unilateral hemispheric infarction in a randomized controlled clinical trial (RCT).

\section{Methods}

\section{Standard protocol approvals, registrations, and patient consents}

The study was reviewed and approved by the Institutional Review Board at Beth Israel Deaconess Medical Center. We confirm that all research procedures was performed in accordance with relevant regulations and in accordance with the Declaration of Helsinki. A written informed consent was obtained for all trial participants or their legal guardians. A Data Safety Monitoring Board with assistance from a Medical Safety Monitor reviewed the progress of the trial. This clinical trial was registered on Clinicaltrials.gov (URL: http://www.clinicaltrials.gov. Unique identifier: NCT01919112) on 8/08/ 2013.

\section{Trial Design and Participants}

FEASt is an early phase-2 double-blind, single-center RCT conducted from September 2013 -September 2019. The rationale, design and protocol of the trial have been published previously [21]. All subjects were recruited at Beth Israel Deaconess Medical Center (BIDMC) in Boston, MA. We enrolled patients with an acute-subacute unilateral hemispheric infarction, day 2-day 6 since the qualifying stroke, between 21-90 years of age, with moderate to severe dysphagia, defined as a Penetration And Aspiration Scale [22] (PAS) score $\geq 4$ on a standardized videofluoroscopic study (VFSS). The main exclusion criteria were pre-stroke swallowing difficulties, severe stroke (NIHSS score $\geq 25$ ), or presence of any other contraindication for tDCS [21].

\section{Randomization and Masking}

Subjects were randomized to 1 of 3 intervention arms-a Low-Dose tDCS, a High-Dose tDCS, or a Sham group, using computer based randomization stratified according to the baseline PAS scores (4-6 versus 7-8). Randomization codes were generated electronically by an independent study Data Coordination Center (DCC). Three individuals, not involved in any other study procedure received confidential electronic notification about the 
randomization codes and programmed the tDCS device accordingly. They also interrogated the device after each session to verify its fidelity to the stimulation allocation. All de-identified VFSS were sent electronically to the Boston University Medical Center laboratory for detailed analysis and results were fed directly to the DCC. These processes ensured complete concealment of intervention allocation and blinding of all investigators and subjects.

\section{Swallowing Procedures and Outcomes Assessments}

All consecutive acute ischemic stroke (AIS) patients admitted to BIDMC were screened for trial participation. All patients received a standardized dysphagia screening using a 3-ounce water swallow test [23] and underwent a swallow evaluation by a Speech-Language pathologist (SLP) if suspected of having dysphagia. Those with moderate to severe dysphagia (PAS $\geq 4$ ) on a standardized VFSS evaluation were enrolled if they fulfilled all study criteria. The VFSS protocol employed 3 consistencies (nectar, pudding and thin liquids) and 5 swallows at trial enrollment (baseline) and after the 10th or last stimulation session (post-stimulation). The arithmetic mean of the PAS score was computed for each subject (composite PAS score) at these time points. A Functional Oral Intake Scale (FOIS) score [24] was computed by the study SLP at baseline, after 5th and final session; in addition, at 30days after the index stroke, an investigator, blinded to the intervention allocation, collected a FOIS score over the phone or in-person using a standardized questionnaire with the subject or a legally authorized representative familiar with subject's current dietary status. An investigator certified in the performance of the NIH Stroke Scale (NIHSS) [25] assigned scores for each participant at enrollment, after every second stimulation sessions and after the last session. All subjects underwent a brain MRI with diffusion weighted imaging (DWI) prior to enrollment, which were used to create a binary map of the acute stroke lesion and construct a novel radiological variable, the corticobulbar tract-lesion load (CBT-LL) (below).

The primary efficacy outcome was a change in PAS scores based on VFSS [22] from baseline to post-stimulation. The primary safety outcomes were incidence of seizures, deterioration in global neurological functions ( $\geq 4$-point increase in the total NIHSS score), motor functions ( $\geq 2$ points increase in the motor sub-item of the NIHSS score on the same limb), swallowing functions (increase in PAS score by $\geq 2$ points compared to baseline), and stroke specific mortality during the period of active stimulation. The main secondary efficacy outcomes were improvement in diet as assessed by a change in FOIS scores [24] at 30-days post-stimulation and changes in swallowing physiology parameters on VFSS from baseline to post-stimulation [pharyngeal delay time (PDT), pharyngeal constriction ratio (PCR), and hyoid, laryngeal and pharyngeal excursion (HLPE)] [21] [26-28].

The CBT-LL variable was derived by creating a canonical tract of the corticobulbar tract in spatially standardized space using the swallowing related cortical fMRI activation and the posterior pons as seed regions. The fMRI experiment consisted of repeated swallowing trials contrasted with whole hand opening and closing tasks, done at the same frequency as the swallowing tasks. The fMRI sequences were acquired with a gradient-echo T2*-weighted MR pulse sequence using our own modification of a sparse temporal sampling method with clustered volume acquisition to overcome imaging artifacts caused by swallowing motion. The voxel clusters of significant cortical activation were used as a seed region (to identify the cortical origin of the CBT) with the second seed region in the posterior pons. The DTI scans were high resolution studies obtained in 12 healthy elderly controls. Image acquisition, analysis, construction of corticobulbar tracts and computation of CBT-lesion load were performed as previously described by Zhu et al [29]. Overlaying the manually drawn lesion maps derived from DWI sequences of trial participants onto the canonical CBT allowed us to calculate a CBT-lesion-load variable. For clinical interpretability, the CBT-LL variable was dichotomized for each subject based on their volume, into 2 groups (< group median, $\geq$ group median) to assess for effect modification of this variable on the experimental intervention. 
We performed a post-hoc analysis using a FOIS score threshold $\geq 5$ as clinically meaningful improvement in dietary intake based on a recent noteworthy publication that utilized this threshold to develop an instrument for prognosticating dysphagia recovery [30]. This cut-off corresponds to a dietary intake of a single consistency or worse and demonstrated to correlate with significant reduction in protein, energy and fluid intake, and suggested as an indication for gastrostomy tube placement after stroke [31-33].

\section{Experimental Intervention}

The stimulation session were performed for 20 minutes twice daily over 5 consecutive days. Anodal tDCS ( 2 mA) or sham was delivered via a battery-driven, constant current stimulator (NeuroConn-DC Stimulator Plus) using saline soaked electrodes (anode $3 \times 5 \mathrm{~cm}$; reference electrode $5 \times 7 \mathrm{~cm}$ ). We targeted the healthy swallowing motor cortex using the 10-20 EEG electrode placement system, placing the anode mid-distance between C3/T3 [left] or C4/T4 [right] over the unaffected hemisphere and the reference electrode over the contralateral supraorbital region. We had previously verified the location of the stimulating electrode using a combination of functional brain MRI (fMRI) and anatomical brain MRI scans [21]. The electrode positioning was re-confirmed in a subset of trial participants using anatomical brain MRI scans. The atDCS electric field $(\mathrm{V} / \mathrm{m})$ distribution was modeled using a freely available software package called SimNIBS [34] (Fig. 1). The MNI ICBM $1521 \mathrm{~mm}$ T1 weighted image was used to perform simulation [35]. As shown in Fig. 1, the electric field distribution $(\mathrm{V} / \mathrm{m})$ is influenced by the shape, location, and current applied through the electrodes.

The High-Dose tDCS group received 2mA atDCS twice daily for a total of 20 minutes (total charge density 16 $\mathrm{C} / \mathrm{cm} 2$ ); Low-Dose group received $2 \mathrm{~mA}$ alternating with sham stimulation daily for a total of 20 minutes (total charge density $8 \mathrm{C} / \mathrm{cm} 2$ ) and Sham group received sham stimulation twice daily.

All stimulation sessions were conducted concurrently with standardized effortful swallowing exercises [36]. All study SLPs and investigators were trained by an expert (SL) on eliciting these maneuvers. Occurrence of an effortful swallow was verified by a laryngeal microphone attached to the patient's throat. We aimed to obtain 40 effortful swallows during each session, which were recorded and entered into the study website.

\section{Statistical Analysis}

We planned to randomize 99 subjects- 33 in each arm, estimated to detect a difference of 1.0 and 1.15 standard deviations (SD) between groups in the mean primary outcome measure with a type I error rate of $2.5 \%$ and power $80 \%$ and $90 \%$, respectively.

The primary efficacy analysis was performed in an intent-to-treat approach. Our outcome variable was a change in the arithmetic mean PAS scores (averaged for 5 different swallows at each time point), before and after intervention and compared across groups. We used a linear model using PROC GLM in SAS to analyze the mean change in PAS scores with the intervention after adjusting for baseline NIHSS scores and age, which were included as covariates based on clinical considerations after screening for other confounders. Similar analyses were performed on the dietary outcome (mean change in FOIS scores at 30-days) and other secondary analysis on changes in swallowing physiology (PDT, PCR, and HLPE). The incidence of safety outcomes were captured as a whole and compared separately across groups. Intraclass correlation (ICC) for PAS scores was performed on a subset of VFSS (randomly selected 303 swallow evaluations) between 2 reviewers (JP and SL). Regression models using interaction terms were used to assess for heterogeneity of intervention effects across the CBT-LL. 
In addition, we conducted the following post-hoc analysis: 1) PRESS score, a recently validated prognostic tool for spontaneous swallowing recovery after an AIS, was computed for each participant and incorporated in an adjusted analysis for the efficacy outcomes [30]; 2) the total number of experimental sessions (10 or < 10) for every subject was used as a covariate in an adjusted analysis for our primary outcome; 3) Fisher-exact test was conducted to compare the proportion of subjects with a FOIS score $\geq 5$ in the combined atDCS group versus sham. Treatment comparison p-values were considered statistically significant at the two-sided 0.05 level of significance; interaction p-values were considered statistically significant at the 0.15 level of significance given the relatively low power for tests of interaction to detect a true interaction. SAS Version 9.4 was used to carry out all analyses.

\section{Results}

A total of 328 subjects were screened for the trial and 42 were enrolled [ 25 female, 17 male; mean age 71 years (SD, 13.2)] (Fig. 2) (Table 1).The cohort had moderately severe strokes at study entry (mean NIHSS score 12.3; SD, 5.2). There were 3 deaths (one per arm) and 1 subject was lost to the 30 -day follow-up. Overall 18 (43\%) subjects completed all 10 sessions and 41 (98\%) completed at least 5 stimulation sessions. Forty effortful swallows were completed in 291/ 382 (76\%) stimulation sessions. No subject withdrew consent or stopped stimulation due to discomfort; 38 (90\%) completed a 30-day follow-up. There were no major protocol violations. 
Table 1

Baseline Characteristics of Trial Cohort in Sham and Anodal Transcranial Direct Stimulation (atDCS) Groups. (aNational Institute of Health Stroke Scale; b- Penetration and Aspiration Scale; c- Functional Oral Intake Scale).

\begin{tabular}{|c|c|c|c|}
\hline Characteristics & $\begin{array}{l}\text { Sham } \\
N=15\end{array}$ & $\begin{array}{l}\text { Low-Dose } \\
\text { atDCS } \\
\mathrm{N}=13\end{array}$ & $\begin{array}{l}\text { High-Dose } \\
\text { atDCS } \\
\mathrm{N}=14\end{array}$ \\
\hline $\begin{array}{l}\text { Age } \\
\text { Mean (SD) }\end{array}$ & $73(14.1)$ & $72(13.3)$ & $68(12.6)$ \\
\hline Sex & $9(60.0)$ & $5(38.5)$ & $11(78.6)$ \\
\hline $\begin{array}{l}\text { No. (\%)Female } \\
\text { No. (\%) Male }\end{array}$ & $6(40)$ & $8(61.5)$ & $3(21.4)$ \\
\hline Race & $14(93.3)$ & $11(84.6)$ & $9(64.3)$ \\
\hline No. (\%) White & 0 & $2(15.4)$ & $3(21.4)$ \\
\hline No. (\%) Black & 0 & 0 & $2(14.3)$ \\
\hline $\begin{array}{l}\text { No. (\%) Asian } \\
\text { No. (\%) Unknown }\end{array}$ & $1(6.7)$ & 0 & 0 \\
\hline NIHSS ${ }^{a}$ Score (SD) & $11.5(5.0)$ & $12.5(6.1)$ & $12.8(4.7)$ \\
\hline PAS ${ }^{b}$ Score (SD) & $4(1.5)$ & $4.1(1.1)$ & $4(1.2)$ \\
\hline FOIS $^{c}$ Score (SD) & $2.7(1.3)$ & $2.8(1.5)$ & $3.3(1.6)$ \\
\hline Stroke Laterality & $9(60)$ & $6(46.2)$ & $3(21.4)$ \\
\hline No. (\%) Right Hemisphere & $6(40)$ & $7(53.8)$ & $11(78.6)$ \\
\hline No. (\%) Left Hemisphere & & & \\
\hline $\begin{array}{l}\text { Duration of Stroke Symptoms at Enrollment in Hours } \\
\text { (SD) }\end{array}$ & $\begin{array}{l}84.9 \\
(39.6)\end{array}$ & $98.4(35.4)$ & $78.0(33.1)$ \\
\hline No. (\%) Previous Stroke & $2(13.3)$ & $2(15.4)$ & $1(7.1)$ \\
\hline No. (\%) Hypertension & $12(80)$ & $9(69.2)$ & $10(71.4)$ \\
\hline No. (\%) Diabetes & $6(40)$ & $4(30.8)$ & $4(28.6)$ \\
\hline No. (\%) Atrial Fibrillation & $3(20)$ & $3(23.1)$ & $7(50.0)$ \\
\hline No. (\%) Coronary Artery Disease & $2(13.3)$ & $3(23.1)$ & $4(28.6)$ \\
\hline
\end{tabular}

No seizures, deterioration in global neurological, motor or swallowing functions were observed in any arm. Overall, 7 unanticipated serious adverse events occurred that were adjudicated as being unrelated to trial procedures (Table 2). 
Table 2

Serious Adverse Events Summary.

\begin{tabular}{|c|c|c|c|c|c|c|c|c|}
\hline & \multicolumn{2}{|c|}{$\begin{array}{l}\text { Sham }(\mathrm{N}= \\
15)\end{array}$} & \multicolumn{2}{|c|}{$\begin{array}{l}\text { Low-Dose tDCS (N } \\
=13)\end{array}$} & \multicolumn{2}{|c|}{$\begin{array}{l}\text { High-Dose tDCS (N } \\
=14)\end{array}$} & \multicolumn{2}{|c|}{$\begin{array}{l}\text { Overall }(\mathrm{N}= \\
42)\end{array}$} \\
\hline & $\mathbf{N}$ & $\%$ & $\mathbf{N}$ & $\%$ & $\mathbf{N}$ & $\%$ & $\mathbf{N}$ & $\%$ \\
\hline \multirow[t]{2}{*}{ At Least One Serious Adverse Event } & 4 & 27 & 1 & 8 & 2 & 14 & 7 & 17 \\
\hline & 0 & 0 & 0 & 0 & 0 & 0 & 0 & 0 \\
\hline Maximum Severity Experienced & 0 & 0 & 0 & 0 & 0 & 0 & 0 & 0 \\
\hline Mild & 1 & 7 & 0 & 0 & 1 & 7 & 2 & 5 \\
\hline Moderate & 1 & 7 & 0 & 0 & 0 & 0 & 1 & 2 \\
\hline Severe & 1 & 7 & 0 & 0 & 0 & 0 & 1 & 2 \\
\hline Life Threatening/Disabling & 0 & 0 & 0 & 0 & 0 & 0 & 0 & 0 \\
\hline Fatal & 1 & 7 & 1 & 8 & 1 & 7 & 3 & 7 \\
\hline Related to Stroke & 1 & 100 & 1 & 100 & 1 & 100 & 3 & 100 \\
\hline \multirow[t]{2}{*}{ Not Related to Stroke } & 0 & 0 & 0 & 0 & 0 & 0 & 0 & 0 \\
\hline & 0 & 0 & 0 & 0 & 0 & 0 & 0 & 0 \\
\hline $\begin{array}{l}\text { Maximum Relationship to Study } \\
\text { Intervention }\end{array}$ & 4 & 27 & 1 & 8 & 2 & 14 & 7 & 17 \\
\hline Unrelated & 3 & 75 & 1 & 100 & 2 & 100 & 6 & 86 \\
\hline Unlikely & 0 & 0 & 0 & 0 & 0 & 0 & 0 & 0 \\
\hline Possible & 1 & 25 & 0 & 0 & 0 & 0 & 1 & 14 \\
\hline Probable & 0 & 0 & 0 & 0 & 0 & 0 & 0 & 0 \\
\hline Definite & 0 & 0 & 0 & 0 & 0 & 0 & 0 & 0 \\
\hline
\end{tabular}

The relevant parameters for our primary efficacy outcomes are summarized in Table 3. The baseline PAS for all groups combined $(n=42)$ were approximately normally distributed. No significant differences in change in PAS scores between groups were seen. Adjusting for baseline PAS, NIHSS scores and age, mean [standard error, (SE)] change in PAS scores were - 0.96 (0.33) in sham, -0.81 (0.36) in Low-Dose and - 0.34 (0.35) in High-Dose; pairwise differences $(p>0.40)$. In the post-hoc analysis adjusting for baseline PRESS score, the mean changes in PAS scores were - $0.97(0.34)$ in sham, $-0.83(0.36)$ in Low-Dose and $-0.31(0.36)$ in High-Dose; pairwise differences $(p>0.40)$. The mean change in PAS scores between subjects completing 10 sessions $(n=18)$ versus those who did not $(n=$ 24), were - 0.99 (SD 1.44) and - 0.49 (SD 1.41), respectively; after adjustment for age, baseline NIHSS and PAS scores, the least square estimate of the difference between group mean was -0.57 ( $S E \pm 1.41), p=0.22$. The ICC of the raw PAS scores on randomly selected 303 swallow evaluations between the 2 evaluators was 0.67 . 
Table 3

Primary Efficacy Outcome-Intent to Treat Analysis. Changes in the Penetration-Aspiration Scale (PAS) Scores Across Sham and Anodal Transcranial Direct Stimulation (atDCS) Groups.

\begin{tabular}{|llll|}
\hline Characteristic & Sham & Low-Dose atDCS & High-Dose atDCS \\
\hline Baseline PAS Score & 15 & 13 & 14 \\
$\mathrm{~N}$ & $4(1.6)$ & $4.1(1.0)$ & $4.1(1.1)$ \\
Mean (SD) & $2.4,8.0$ & $1.6,5.5$ & $2.4,6.4$ \\
Maximum, Minimum & & & \\
Exit PAS Score & 15 & 13 & 14 \\
$\mathrm{~N}$ & $3.2(1.6)$ & $3.3(1.4)$ & $3.7(1.5)$ \\
Mean (SD) & $1.0,5.4$ & $1.2,7.3$ & $1.2,6.8$ \\
Maximum, Minimum & & & 14 \\
Change in PAS Score & 15 & 13 & $-0.4(1.2)$ \\
$\mathrm{N}$ & $-0.8(1.6)$ & $-0.8(1.5)$ & $-2.2,1.6$ \\
Mean (SD) & $-4.3,1.3$ & $-3.7,2.5$ & \\
Maximum, Minimum & & & \\
\hline
\end{tabular}

The relevant parameters for our dietary outcome are outlined in Table 4. There were no significant differences in baseline FOIS scores between groups. After adjusting for baseline FOIS, NIHSS scores and age, mean (SE) change in FOIS scores were 2.07 (0.35) in Sham, 2.46 (0.38) in Low-Dose and 3.05 (0.38) in High-Dose (pairwise p >0.15). A post-hoc analysis of FOIS scores at 30-days showed that 22 /24 subjects in the combined tDCS group had a FOIS score $\geq 5$ compared to $8 / 14$ in sham ( $p=0.037$, Fisher-exact). 
Table 4

Dietary Outcome-Intent to Treat Analysis. Changes in the Functional Oral Intake Scale (FOIS) Scores Across Sham and Anodal Transcranial Direct Stimulation (atDCS) Groups.

\begin{tabular}{|llll|}
\hline Characteristic & Sham & Low-Dose atDCS & High-Dose atDCS \\
\hline Baseline FOIS Score & 15 & 13 & 14 \\
$\mathrm{~N}$ & $2.7(1.3)$ & $2.8(1.5)$ & $3.3(1.6)$ \\
Mean (SD) & 1,6 & 1,6 & 1,5 \\
Maximum, Minimum & & & \\
Exit FOIS Score & 14 & 12 & 12 \\
$\mathrm{~N}$ & $4.9(2.2)$ & $5.4(0.9)$ & $6.3(1.0)$ \\
Mean (SD) & 1,7 & 4,7 & 4,7 \\
Maximum, Minimum & & & 12 \\
Change in FOIS Score & 14 & 12 & $2.9(1.2)$ \\
$\mathrm{N}$ & $2.1(1.7)$ & $2.5(1.7)$ & 1,5 \\
Mean (SD) & 0,5 & 0,6 & \\
Maximum, Minimum & & & \\
\hline
\end{tabular}

The secondary outcomes for pre-specified swallowing physiological outcomes are summarized in Table 5. No statistically significant difference between intervention groups were seen in any of the pre-defined physiological parameters in adjusted models that included baseline NIHSS score and age. An a priori assessment for effect modification across CBT-LL on the trial intervention showed heterogeneity of intervention effects for the PAS ( $p=$ 0.116 ) and FOIS $(p=0.017)$ outcomes (Table 6). In patients below median CBT-LL, the mean change in PAS score was smallest for Sham and largest for High-Dose tDCS, whereas in patients at or above median CBT-LL, the largest mean change in PAS score was seen for Sham. On the contrary, in patients below median CBT-LL, the mean increase in FOIS score was similar across the three groups, whereas in patients at or above median CBT-LL, the mean increase in FOIS score was smallest for Sham and largest for High-Dose tDCS. This suggests that CBT-LL can potential identify responders versus potential responders using FOIS as an outcome measure. 
Table 5

Swallowing Physiology Outcomes Across Sham and Anodal Transcranial Direct Stimulation (atDCS) Groups. (a- Pharyngeal Constriction Ratio; *b-Pharyngeal Delay Time)

\begin{tabular}{|c|c|c|c|}
\hline Characteristic & Sham & Low-Dose atDCS & High-Dose atDCS \\
\hline Baseline PCR & 15 & 13 & 14 \\
\hline $\mathrm{N}$ & $0.12(0.11)$ & $0.13(0.15)$ & $0.08(0.07)$ \\
\hline Mean (SD) & $0.02,0.45$ & $0.01,0.54$ & $0.01,0.28$ \\
\hline \multicolumn{4}{|l|}{ Minimum, Maximum } \\
\hline Change from Baseline to Day 5 PCR & 13 & 13 & 14 \\
\hline $\mathrm{N}$ & $-0.02(0.06)$ & $-0.1(0.12)$ & $-0.01(0.09)$ \\
\hline Mean (SD) & $-0.09,0.09$ & $-0.29,0.05$ & $-0.16,0.19$ \\
\hline \multicolumn{4}{|l|}{ Minimum, Maximum } \\
\hline Baseline PDT ${ }^{b}$ & 14 & 11 & 13 \\
\hline $\mathrm{N}$ & $13.3(14.9)$ & $28.2(29.3)$ & $11.2(16.1)$ \\
\hline Mean (SD) & $0,42.8$ & $0,88.3$ & $0,60.0$ \\
\hline \multicolumn{4}{|l|}{ Minimum, Maximum } \\
\hline Change from Baseline to Day 5 PDT & 14 & 11 & 13 \\
\hline $\mathrm{N}$ & $0.83(20.6)$ & $-7.3(43.4)$ & $5.2(21.3)$ \\
\hline Mean (SD) & $-37.2,43.3$ & $-75,73.3$ & $-41.7,43.3$ \\
\hline \multicolumn{4}{|l|}{ Minimum, Maximum } \\
\hline Baseline Hyoid Excursion & 15 & 12 & 13 \\
\hline $\mathrm{N}$ & $1.08(0.44)$ & $1.19(0.31)$ & $1.13(0.58)$ \\
\hline Mean (SD) & $0.39,1.86$ & $0.64,1.65$ & $0.27,2.03$ \\
\hline \multicolumn{4}{|l|}{ Minimum, Maximum } \\
\hline Change from Baseline to Day 5 Hyoid Excursion & 12 & 12 & 13 \\
\hline $\mathrm{N}$ & $0.05(0.87)$ & $-0.2(0.36)$ & $-0.4(0.75)$ \\
\hline Mean (SD) & $-1.36,1.46$ & $-0.66,0.54$ & $-1.72,1.40$ \\
\hline \multicolumn{4}{|l|}{ Minimum, Maximum } \\
\hline Baseline Laryngeal Excursion & 12 & 9 & 12 \\
\hline $\mathrm{N}$ & $1.98(0.53)$ & $2.07(1.0)$ & $2.04(0.77)$ \\
\hline Mean (SD) & $1.24,3.13$ & $0.35,3.48$ & $0.75,3.47$ \\
\hline Minimum, Maximum & & & \\
\hline
\end{tabular}




\begin{tabular}{|llll|}
\hline Characteristic & Sham & Low-Dose atDCS & High-Dose atDCS \\
\hline Change from Baseline to Day 5 Laryngeal Excursion & 9 & 8 & 12 \\
N & $0.14(0.67)$ & $0.17(1.4)$ & $0.07(0.81)$ \\
Mean (SD) & $-1.08,1.20$ & $-1.21,2.61$ & $-1.12,1.48$ \\
Minimum, Maximum & & & \\
\hline
\end{tabular}

Table 5

Swallowing Physiology Outcomes Across Sham and Anodal Transcranial Direct Stimulation (atDCS) Groups. (aPharyngeal Constriction Ratio; b-Pharyngeal Delay Time)-continued

\begin{tabular}{|llll|}
\hline Characteristic & Sham & Low-Dose atDCS & High-Dose atDCS \\
\hline Baseline Pharyngeal Excursion & 14 & 10 & 13 \\
$\mathrm{~N}$ & $1.17(0.97)$ & $1.22(0.62)$ & $0.95(1.12)$ \\
Mean (SD) & $-0.82,3.13$ & $0.11,2.21$ & $-0.54,3.22$ \\
Minimum, Maximum & & & 13 \\
Change from Baseline to Day 5 Pharyngeal Excursion & 13 & 10 & $0.09(1.10)$ \\
N & $-0.07(0.95)$ & $0.0(1.02)$ & $-1.42,1.89$ \\
Mean (SD) & $-1.24,1.64$ & $-1.46,2.25$ & \\
Minimum, Maximum & & & \\
\hline
\end{tabular}

Table 6

Descriptive Statistics of Penetration-Aspiration Scale (PAS) and Functional Oral Intake Scale (FOIS) with Dichotomized Corticobulbar tract-Lesion Load (CBT-LL).

\begin{tabular}{|c|c|c|c|c|c|c|c|}
\hline \multirow[t]{2}{*}{ Characteristics } & \multicolumn{2}{|l|}{ Sham } & \multicolumn{2}{|c|}{ Low-Dose tDCS } & \multicolumn{3}{|c|}{ High-Dose tDCS } \\
\hline & $\begin{array}{l}\text { CBT-LL } \\
<\text { Median }\end{array}$ & $\begin{array}{l}\text { CBT-LL } \\
\geq \text { Median }\end{array}$ & $\begin{array}{l}\text { CBT-LL } \\
<\text { Median }\end{array}$ & $\begin{array}{l}\text { CBT-LL } \\
\geq \text { Median }\end{array}$ & $\begin{array}{l}\text { CBT-LL } \\
\text { <Median }\end{array}$ & $\begin{array}{l}\text { CBT-LL } \\
\geq \text { Median }\end{array}$ & $\begin{array}{l}\text { p-value for } \\
\text { interaction }\end{array}$ \\
\hline $\begin{array}{l}\text { Mean (SD) } \\
\text { Baseline PAS }\end{array}$ & $4.40(1.79)$ & $3.63(1.46)$ & $4.57(0.67)$ & $3.86(1.11)$ & $3.79(0.99)$ & $4.53(1.29)$ & \\
\hline $\begin{array}{l}\text { Mean (SD) } \\
\text { Change in PAS }\end{array}$ & $\begin{array}{l}-0.46 \\
(1.49)\end{array}$ & $-1.3(1.78)$ & $\begin{array}{l}-0.77 \\
(2.26)\end{array}$ & $\begin{array}{l}-0.80 \\
(0.97)\end{array}$ & $\begin{array}{l}-0.94 \\
(1.03)\end{array}$ & $\begin{array}{l}0.13 \\
(1.23)\end{array}$ & 0.116 \\
\hline $\begin{array}{l}\text { Mean (SD) } \\
\text { Baseline FOIS }\end{array}$ & 2.75(1.39) & $2.71(1.38)$ & $2.00(1.22)$ & $3.25(1.58)$ & $3.75(1.58)$ & $2.67(1.51)$ & \\
\hline $\begin{array}{l}\text { Mean (SD) } \\
\text { Change in } \\
\text { FOIS }\end{array}$ & $3.00(1.83)$ & $1.29(1.25)$ & $3.25(2.06)$ & $2.13(1.46)$ & 2.75(1.04) & $3.25(1.50)$ & 0.017 \\
\hline
\end{tabular}

\section{Discussion}

Results from the FEASt trial show that application of atDCS in the acute-subacute stroke phase to the undamaged cerebral hemisphere is safe and well-tolerated within the stimulation parameters used in this investigation. Although the safety of atDCS in AIS patients has been previously reported in small pilot and a recent larger single- 
center clinical trial [20,37], the FEASt trial is the first investigation to systematically analyze the safety and tolerability of higher doses of atDCS in this stroke phase.

Our experience validates the feasibility of our approach, where subjects with significant neurological and cognitive impairments in the immediate aftermath of a stroke were able to actively participate in this intervention as a protocol requirement over several sessions. We found a high compliance with the experimental swallowing protocol in this trial. Effortful swallowing maneuvers were successfully elicited in all sessions and most participants were able to perform the pre-specified 40 effortful swallows in 20 minutes. A significant proportion of subjects did not complete all 10 sessions primarily due to early hospital discharge based on clinical considerations. The mean change in PAS scores in patients completing 10 sessions was higher than those who did not but this difference did not attain statistical significance in an adjusted analysis.

Although swallowing is a multifaceted process, we chose changes in the risk of aspiration as our primary outcome, using an 8-point ordinal PAS scores, as aspiration leads to dietary restrictions, increases the risk of pneumonia and need for tube feeding. Scoring for PAS was done blindly by investigators with expertise in its interpretation though their ICC was sub-optimal and at par with other studies in the field [38,39]. Several factors may have contributed to the variability in scoring. PAS scores assignments are based on multiple considerations including the depth of airway invasion and the patient's response to it; a recent systematic review shows that misclassification of PAS levels in dysphagia research is common $[39,40]$. Other investigators have highlighted issues with its construct validity and statistical constraints [38]. Our use of a composite PAS score instead of scoring only on thin consistencies may have also decreased the sensitivity to capture aspiration events. Furthermore, our cohort included stroke subjects with moderate to severe disabilities and their compliance with swallowing instructions may have been additional sources of variability. These considerations place some uncertainty around our results which overall did not show a significant difference between groups for our primary efficacy outcome. This early trial experience highlights some of the limitations of using PAS as an outcome instrument for confirmatory clinical trials in stroke related dysphagia.

We included physiological swallowing outcome measures derived from VFSS since they were anticipated to be more sensitive to changes in swallowing status as well as to elucidate the mechanistic underpinnings for swallowing recovery. These measures included the PCR (a surrogate measure for pharyngeal strength with lower ratio indicating better strength), PDT (a temporal measure for briskness of swallow with lower numbers indicating faster swallow) and HLPE (semi-independent movements that close off the airway and shorten the pharynx) [2628]. While there are normative data on these metrics in normal and chronic stroke populations, similar data in AIS population is lacking. We saw substantial variability in these measures and some estimates were clearly discordant with the clinical swallowing outcomes. Our experience demonstrates the challenges of using these metrics in AIS trials, and emphasizes the need for developing more robust VFSS protocols, as well as optimization of analytical methods before they can be reliably deployed in multicenter-RCTs.

The overall objective of dysphagia therapies is to safely restore a normal diet. Unlike, PAS, which primarily assesses pharyngeal and laryngeal events during swallowing, changes in diet, FOIS scores can provide a more holistic assessment of swallowing functions. FOIS is a functional outcome that provides a validated measure of dietary level, is sensitive to changes in swallowing functions and has been tested and validated in stroke population [24]. A theoretical criticism of this scale has been that patients may advance their diet despite persistent swallowing impairment and professional recommendations. We believe this is less likely to have influenced our result. All subjects were randomized and had been clinically stable at their respective dietary levels, the assessors were

Page $13 / 20$ 
blinded and computed the scores based on a structured interview. Higher doses of atDCS were associated with higher FOIS scores but group differences were not statistically significant though this comparison may have been underpowered. A post-hoc analysis for minimal clinically important difference (MCID) in dietary intake using a Fisher-exact test (which yields substantially conservative levels of statistical significance), revealed significantly better intake at 30-days in the combined atDCS compared to sham group [41].

Dysphagic stroke patients usually have more severe deficits and overall fare poorly compared to their nondysphagic counterparts $[1,5,9]$. We expected that other factors might influence the response to our intervention [42]. We used CBT-LL to better understand the variability in response and found a significant heterogeneity of intervention effect across this variable. These findings are hypothesis generating but suggest that the effects of atDCS are primarily intracortical and its effects on swallowing behavior can be influenced by the extent of damage to the corticobulbar tracts with more damage to one CBT making the contribution and modulation of activity in the other intact hemisphere more important.

\section{Limitations}

A significant limitation was accruement of lower than anticipated sample size during the funding period that may have affected the power to detect our efficacy endpoints. Analyses of VFSS based outcomes showed significant variability and our main outcome PAS had suboptimal ICC, which may have undermined our ability to detect a real change. Future trials will require a better selection of swallowing parameters as well as further optimization in their acquisition and analysis. It will also need validated metrics to identify minimal clinically important differences (MCID) in meaningful change in swallowing functions. There was suggestion of a dose effect reflected in some of the swallowing measures but not in others. This needs further corroboration and exploration of other doses that may be more effective.

\section{Conclusion}

The results of FEASt demonstrate that application of atDCS to the unaffected hemisphere paired with swallowing exercises in acute stroke patients with post-stroke dysphagia is safe and feasible though it did not decrease the risk of aspiration events in this early phase-2 RCT. It improved dietary intake, which needs further validation in a larger trial. A recent single-center trial demonstrated that $1 \mathrm{~mA}$ of daily tDCS significantly improved swallowing function in AIS patients [37]. Meta-analyses from studies on motor recovery have suggested a dose-effect relationship although only studies up to $2 \mathrm{~mA}$ were included this metanalysis [43]. More recent studies have revealed that higher dose tDCS $(4 \mathrm{~mA})$ is feasible, well tolerated, safe, and leads to stronger activity changes $[44,45]$. Future trials should consider modifications in stimulation parameters including alternate atDCS doses and use of clinically meaningful swallowing outcomes. The heterogeneity of intervention effects across CBT-lesion load observed in this trial merits further investigation and can be used to stratify subject enrollment and analysis. Our trial experience highlights challenges but also the promise of this technique in improving stroke related dysphagia. Lessons drawn from the FEASt trial can help lay the foundation for a larger confirmatory-RCT for improving stroke related dysphagia.

\section{Declarations}

\section{Acknowledgements:}


Funding Support: This trial was funded Grant support from the National Institute on Deafness and Other Communication Disorders of the National Institutes of Health (NIH) under Award Number R01DC012584 (PI: Kumar). GS acknowledges support from NINDS (U01NS102353) and NIMH (R01MH111874). The content of this work is solely the responsibility of the authors and does not necessarily represent the official views of the $\mathrm{NIH}$. The authors do not have any other conflicts of interest to report.

Additional Contributions: We thank the members of the Data Safety Monitoring Board and the Medical Safety Monitor for their oversight and guidance of this trial: Aneesh Singhal MD (Chair); Mark Schactman MHS, MS; Sherry H-Y Chou MD, MMSc; Jayme Dowdall MD; Jordan R. Green PhD; and Shoshana J. Herzig, MD, MPH (MSM). We thank the following members of the Voice, Speech and Swallowing Unit at BIDMC for their trial support and assistance with study related swallowing evaluations: Karen M Sheffler, MS, CCC-SLP; Julie M Gilmer, CCC-SLP; Ana C Garrido White, MS, CCC-SLP; Sarah E MacKenzie, CCC-SLP; Lindsay R Griffin, PhD, CCC-SLP; Elana R Katz, Laina Piera, MS, CCC-SLP; Amanda Warren, MS, CCC-SLP; Brooke Littleton, MS, CCC-SLP. We thank William Pearson, PhD and Gintas Krisciunas, MA, MPH for their insights into development of the trial protocol and plan for execution.

Meeting Presentation: Presented in part at International Stroke Conference, February 20, 2020; Los Angeles, CA and American Neurological Association, October 8, 2020.

Conflicts of Interest: The authors do not have any other conflicts of interest to disclose.

Availability of Data and Materials: All data generated or analysed during this study are included in this published article and its supplementary information files.

\section{References}

1. Martino R, Foley N, Bhogal S, Diamant N, Speechley M, Teasell R. Dysphagia after stroke: incidence, diagnosis, and pulmonary complications. Stroke; a journal of cerebral circulation 2005;36(12):2756-63.

2. Barer $\mathrm{DH}$. The natural history and functional consequences of dysphagia after hemispheric stroke. Journal of neurology, neurosurgery, and psychiatry 1989;52(2):236-41.

3. Gordon C, Hewer RL, Wade DT. Dysphagia in acute stroke. British medical journal 1987;295(6595):411-4.

4. Langmore SE, Terpenning MS, Schork A, Chen Y, Murray JT, Lopatin D, et al. Predictors of aspiration pneumonia: how important is dysphagia? Dysphagia 1998;13(2):69-81.

5. Smithard DG, O'Neill PA, Parks C, Morris J. Complications and outcome after acute stroke. Does dysphagia matter? Stroke 1996;27(7):1200-4.

6. Bath PM, Lee HS, Everton LF. Swallowing therapy for dysphagia in acute and subacute stroke. The Cochrane database of systematic reviews 2018;10:Cd000323.

7. Joundi RA, Saposnik G, Martino R, Fang J, Porter J, Kapral MK. Outcomes among patients with direct enteral vs nasogastric tube placement after acute stroke. Neurology 2018;90(7):e544-e52.

8. Galovic M. Comparing nasogastric and direct tube feeding in stroke: Enteral feeding going down the tube. Neurology 2018;90(7):305-6.

9. Smithard DG, O'Neill PA, England RE, Park CL, Wyatt R, Martin DF, et al. The natural history of dysphagia following a stroke. Dysphagia 1997;12(4):188-93. 
10. Ertekin C, Aydogdu I. Neurophysiology of swallowing. Clinical neurophysiology: official journal of the International Federation of Clinical Neurophysiology 2003;114(12):2226-44.

11. Malandraki GA, Sutton BP, Perlman AL, Karampinos DC, Conway C. Neural activation of swallowing and swallowing-related tasks in healthy young adults: an attempt to separate the components of deglutition. Human brain mapping 2009;30(10):3209-26.

12. Hamdy S, Aziz Q, Rothwell JC, Singh KD, Barlow J, Hughes DG, et al. The cortical topography of human swallowing musculature in health and disease. Nature medicine 1996;2(11):1217-24.

13. Hamdy S, Aziz Q, Rothwell JC, Crone R, Hughes D, Tallis RC, et al. Explaining oropharyngeal dysphagia after unilateral hemispheric stroke. Lancet 1997;350(9079):686-92.

14. Hamdy S, Aziz Q, Rothwell JC, Power M, Singh KD, Nicholson DA, et al. Recovery of swallowing after dysphagic stroke relates to functional reorganization in the intact motor cortex. Gastroenterology 1998;115(5):1104-12.

15. Hamdy S, Aziz Q, Rothwell JC, Hobson A, Thompson DG. Sensorimotor modulation of human cortical swallowing pathways. The Journal of physiology 1998;506 (Pt 3):857-66.

16. Gow D, Rothwell J, Hobson A, Thompson D, Hamdy S. Induction of long-term plasticity in human swallowing motor cortex following repetitive cortical stimulation. Clinical neurophysiology: official journal of the International Federation of Clinical Neurophysiology 2004;115(5):1044-51.

17. Kumar S, Schlaug G. Enhancing swallowing recovery after a stroke by harnessing its bihemispheric organization. Annals of neurology 2018;83(4):658-60.

18. Turney TM, Garraway WM, Whisnant JP. The natural history of hemispheric and brainstem infarction in Rochester, Minnesota. Stroke 1984;15(5):790-4.

19. Kumar S, Wagner CW, Frayne C, Zhu L, Selim M, Feng W, et al. Noninvasive brain stimulation may improve stroke-related dysphagia: a pilot study. Stroke; a journal of cerebral circulation 2011;42(4):1035-40.

20. Pisegna JM, Kaneoka A, Pearson WG, Jr., Kumar S, Langmore SE. Effects of non-invasive brain stimulation on post-stroke dysphagia: A systematic review and meta-analysis of randomized controlled trials. Clinical neurophysiology: official journal of the International Federation of Clinical Neurophysiology 2016;127(1):95668.

21. Marchina S, Schlaug G, Kumar S. Study design for the fostering eating after stroke with transcranial direct current stimulation trial: a randomized controlled intervention for improving Dysphagia after acute ischemic stroke. Journal of stroke and cerebrovascular diseases: the official journal of National Stroke Association 2015;24(3):511-20.

22. Rosenbek JC, Robbins JA, Roecker EB, Coyle JL, Wood JL. A penetration-aspiration scale. Dysphagia 1996;11(2):93-8.

23. Suiter DM, Leder SB. Clinical utility of the 3-ounce water swallow test. Dysphagia 2008;23(3):244-50.

24. Crary MA, Mann GD, Groher ME. Initial psychometric assessment of a functional oral intake scale for dysphagia in stroke patients. Archives of physical medicine and rehabilitation 2005;86(8):1516-20.

25. Brott T, Adams HP, Jr., Olinger CP, Marler JR, Barsan WG, Biller J, et al. Measurements of acute cerebral infarction: a clinical examination scale. Stroke 1989;20(7):864-70.

26. Logemann JA, Pauloski BR, Rademaker AW, Colangelo LA, Kahrilas PJ, Smith CH. Temporal and biomechanical characteristics of oropharyngeal swallow in younger and older men. Journal of speech, language, and hearing research: JSLHR 2000;43(5):1264-74. 
27. Leonard R, Belafsky PC, Rees CJ. Relationship between fluoroscopic and manometric measures of pharyngeal constriction: the pharyngeal constriction ratio. The Annals of otology, rhinology, and laryngology 2006;115(12):897-901.

28. Leonard RJ, Kendall KA, McKenzie S, Goncalves MI, Walker A. Structural displacements in normal swallowing: a videofluoroscopic study. Dysphagia 2000;15(3):146-52.

29. Zhu LL, Lindenberg R, Alexander MP, Schlaug G. Lesion load of the corticospinal tract predicts motor impairment in chronic stroke. Stroke 2010;41(5):910-5.

30. Galovic M, Stauber AJ, Leisi N, Krammer W, Brugger F, Vehoff J, et al. Development and Validation of a Prognostic Model of Swallowing Recovery and Enteral Tube Feeding After Ischemic Stroke. JAMA neurology 2019;76(5):561-70.

31. Whelan K. Inadequate fluid intakes in dysphagic acute stroke. Clin Nutr 2001;20(5):423-8.

32. Vivanti AP, Campbell KL, Suter MS, Hannan-Jones MT, Hulcombe JA. Contribution of thickened drinks, food and enteral and parenteral fluids to fluid intake in hospitalised patients with dysphagia. Journal of human nutrition and dietetics: the official journal of the British Dietetic Association 2009;22(2):148-55.

33. Wirth R, Smoliner C, Jager M, Warnecke T, Leischker AH, Dziewas R, et al. Guideline clinical nutrition in patients with stroke. Experimental \& translational stroke medicine 2013;5(1):14.

34. Saturnino GB, Puonti O, Nielsen JD, Antonenko D, Madsen KH, Thielscher A. SimNIBS 2.1: A Comprehensive Pipeline for Individualized Electric Field Modelling for Transcranial Brain Stimulation. In: Makarov S, Horner M, Noetscher G, editors. Brain and Human Body Modeling: Computational Human Modeling at EMBC 2018, Cham $(\mathrm{CH}) ; 2019$, p. 3-25.

35. Fonov V, Evans AC, Botteron K, Almli CR, McKinstry RC, Collins DL, et al. Unbiased average age-appropriate atlases for pediatric studies. Neuroimage 2011;54(1):313-27.

36. Lazarus C, Logemann JA, Song CW, Rademaker AW, Kahrilas PJ. Effects of voluntary maneuvers on tongue base function for swallowing. Folia phoniatrica et logopaedica: official organ of the International Association of Logopedics and Phoniatrics 2002;54(4):171-6.

37. Suntrup-Krueger S, Ringmaier C, Muhle P, Wollbrink A, Kemmling A, Hanning $U$, et al. Randomized trial of transcranial direct current stimulation for poststroke dysphagia. Annals of neurology 2018;83(2):328-40.

38. Steele CM, Grace-Martin K. Reflections on Clinical and Statistical Use of the Penetration-Aspiration Scale. Dysphagia 2017;32(5):601-16.

39. Hind JA, Gensler G, Brandt DK, Gardner PJ, Blumenthal L, Gramigna GD, et al. Comparison of trained clinician ratings with expert ratings of aspiration on videofluoroscopic images from a randomized clinical trial. Dysphagia 2009;24(2):211-7.

40. Borders JC, Brates D. Use of the Penetration-Aspiration Scale in Dysphagia Research: A Systematic Review. Dysphagia 2019.

41. Lydersen S, Pradhan V, Senchaudhuri P, Laake P. Choice of test for association in small sample unordered $\mathrm{r} x \mathrm{C}$ tables. Statistics in medicine 2007;26(23):4328-43.

42. Kumar S, Doughty C, Doros G, Selim M, Lahoti S, Gokhale S, et al. Recovery of swallowing after dysphagic stroke: an analysis of prognostic factors. Journal of stroke and cerebrovascular diseases: the official journal of National Stroke Association 2014;23(1):56-62.

43. Chhatbar PY, Ramakrishnan V, Kautz S, George MS, Adams RJ, Feng W. Transcranial Direct Current Stimulation Post-Stroke Upper Extremity Motor Recovery Studies Exhibit a Dose-Response Relationship. Brain stimulation

Page $17 / 20$ 
2016;9(1):16-26.

44. Chhatbar PY, Chen R, Deardorff R, Dellenbach B, Kautz SA, George MS, et al. Safety and tolerability of transcranial direct current stimulation to stroke patients - A phase I current escalation study. Brain stimulation 2017;10(3):553-9.

45. Shinde AB, Lerud KD, Munsch F, Alsop DC, Schlaug G. Effects of tDCS dose and electrode montage on regional cerebral blood flow and motor behavior. Neuroimage 2021;237:118144.

\section{Figures}

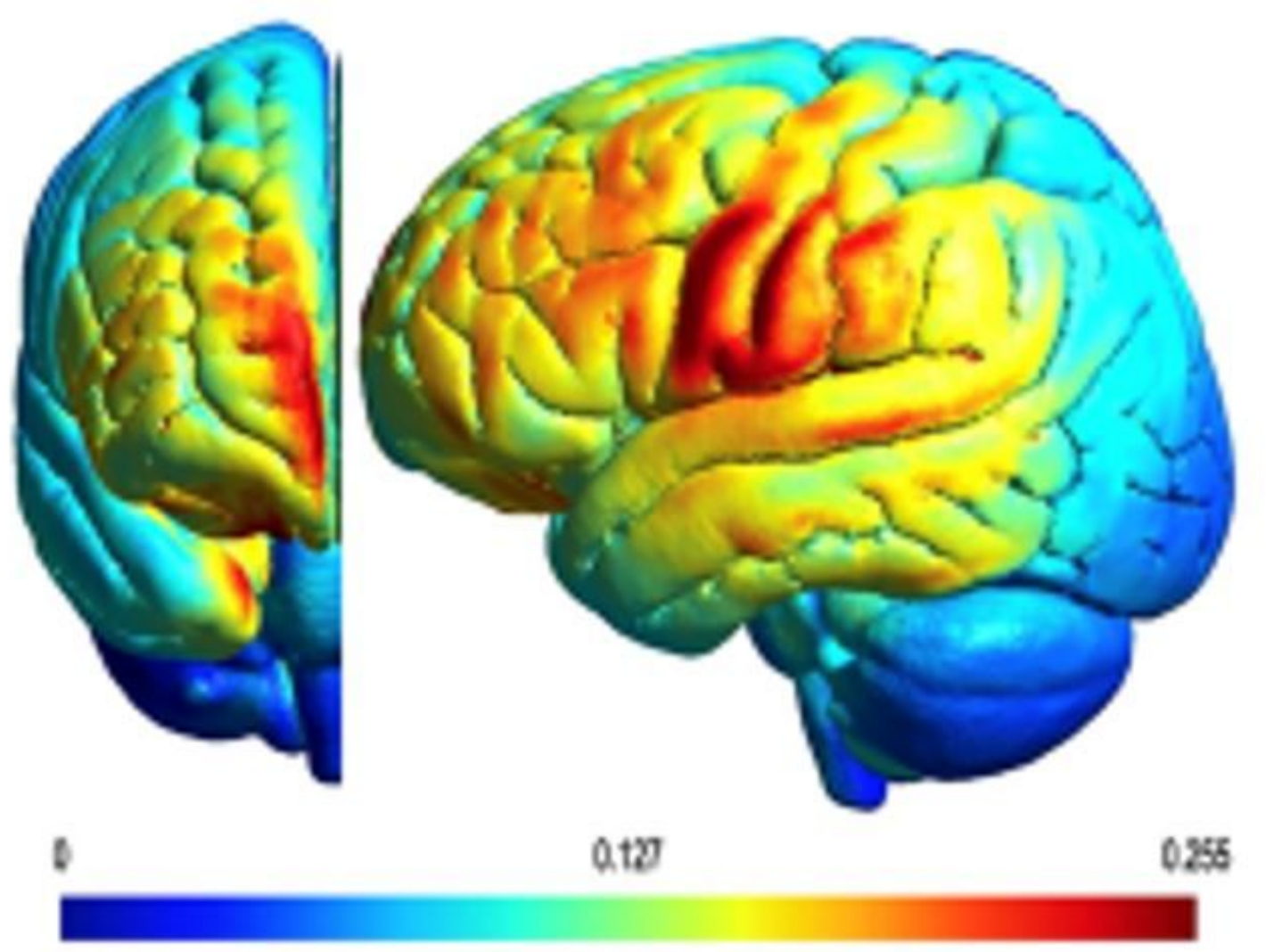

\section{normE}

\section{Figure 1}

The simulated electric field (normal to the cortical surface) with a rectangular anodal electrode of $3 \times 5 \mathrm{~cm}$ positioned with its center at the half-way point between C3 and T3 (left) or C4 and T4 (right) on the unaffected hemisphere respectively, and a rectangular cathodal electrode of $5 \times 7 \mathrm{~cm}$ placed long-way over the surpraorbital region on the affected hemisphere 


\begin{tabular}{|c|c|c|}
\hline & $\begin{array}{l}\text { Assessed for Eligibility } \\
\qquad N=328\end{array}$ & \\
\hline & $\begin{array}{c}\downarrow \\
\text { Randomized } \\
N=42\end{array}$ & $\begin{array}{l}\text { - Excluded } \\
\mathrm{N}=286 \\
\text { - Not meeting trial criteria } \\
\mathrm{N}=214 \\
\text { - Refused to consent } \\
\mathrm{N}=72\end{array}$ \\
\hline $\begin{array}{l}\text { Sham Group } \\
\mathrm{N}=15 \\
\text { - Completed at least } 5 \\
\text { sessions } \\
\mathrm{N}=14 \\
\text { - Completed all } 10 \\
\text { sessions } \\
\mathrm{N}=5 \\
\cdot \text { Completed } 1 \text { month } \\
\text { follow-up } \\
\mathrm{N}=14\end{array}$ & $\begin{array}{l}\text { Low-dose tDCS } \\
\mathrm{N}=13 \\
\text { - Completed at least } 5 \\
\text { sessions } \\
\mathrm{N}=13 \\
\text { - Completed all } 10 \\
\text { sessions } \\
\mathrm{N}=8 \\
\text { - Completed 1-month } \\
\text { follow-up } \\
\mathrm{N}=12\end{array}$ & $\begin{array}{l}\text { High-dose tDCS } \\
\mathrm{N}=14 \\
\text { - Completed at least } 5 \\
\text { sessions } \\
\mathrm{N}=14 \\
\text { - Completed all } 10 \\
\text { sessions } \\
\mathrm{N}=5 \\
\text { - Completed 1-month } \\
\text { follow-up } \\
\mathrm{N}=12\end{array}$ \\
\hline$\downarrow$ & $\downarrow$ & $\downarrow$ \\
\hline $\begin{array}{l}\text { Lost to follow-up } \\
\qquad \begin{array}{l}\mathrm{N}=1 \\
\text { (death) }\end{array}\end{array}$ & $\begin{array}{l}\text { Lost to follow-up } \\
\qquad \begin{array}{l}\mathrm{N}=1 \\
\text { (death) }\end{array}\end{array}$ & $\begin{array}{l}\text { Lost to follow-up } \\
\qquad \mathrm{N}=2 \\
\text { (death, unable to } \\
\text { locate) }\end{array}$ \\
\hline$\downarrow$ & $\downarrow$ & $\downarrow$ \\
\hline $\begin{array}{l}\text { Included in Analysis } \\
\cdot N=15\left(\text { PAS }^{\mathrm{a}}\right) \\
\cdot \mathrm{N}=14\left(\mathrm{FOIS}^{\mathrm{b}}\right) \\
\cdot \mathrm{N}=13\left(\mathrm{PCR}^{\mathrm{C}}\right) \\
\cdot \mathrm{N}=14\left(\mathrm{PDT}^{\mathrm{d}}\right) \\
\cdot \mathrm{N}=12\left(\mathrm{HE}^{\mathrm{e}}\right) \\
\cdot \mathrm{N}=9\left(\mathrm{LE}^{\mathrm{f}}\right) \\
\cdot \mathrm{N}=13\left(\mathrm{PE}^{\mathrm{g}}\right)\end{array}$ & $\begin{array}{l}\text { Included in Analysis } \\
\cdot \mathrm{N}=13 \text { (PAS) } \\
\cdot \mathrm{N}=12 \text { (FOIS) } \\
\cdot \mathrm{N}=13(\mathrm{PCR}) \\
\cdot \mathrm{N}=11(\mathrm{PDT}) \\
\cdot \mathrm{N}=12(\mathrm{HE}) \\
\cdot \mathrm{N}=8(\mathrm{LE}) \\
\cdot \mathrm{N}=10(\mathrm{PE})\end{array}$ & $\begin{array}{l}\text { Included in Analysis } \\
\cdot N=14 \text { (PAS) } \\
\cdot N=12 \text { (FOIS) } \\
\cdot N=14(\text { PCR) } \\
\cdot N=13(\text { PDT) } \\
\cdot N=13(\mathrm{HE}) \\
\cdot N=12 \text { (LE) } \\
\cdot N=13(\text { PE) }\end{array}$ \\
\hline
\end{tabular}

Figure 2

Consort Flow Diagram Showing Progress of Subjects in the Trial Sham and Anodal Transcranial Direct Stimulation (atDCS) Groups. (a-Penetration and Aspiration Scale; b-Functional Oral Intake Scale; c-Pharyngeal Constriction Ratio; d- Pharyngeal Delay Time; e-Hyoid Excursion; f-Laryngeal Excursion; g-Pharyngeal Excursion).

\section{Supplementary Files}

This is a list of supplementary files associated with this preprint. Click to download.

- FEAStSupplementAdverseevents.docx 
- FEAStCLOSEDDSMBtables2019Mar20.xls

- TrialProtocol.docx 